\title{
Probing the location of the substrate binding site of ascorbate oxidase near type 1 copper: an investigation through spectroscopic, inhibition and docking studies
}

\author{
Laura Santagostini $^{\mathrm{a}}$, Michele Gullotti ${ }^{\mathrm{a}}$, Luca De Gioia ${ }^{\mathrm{b}}$, Piercarlo Fantucci ${ }^{\mathrm{b}}$, \\ Elena Franzini ${ }^{b}$, Augusto Marchesini ${ }^{c}$, Enrico Monzani ${ }^{\mathrm{d}}$, Luigi Casella ${ }^{\mathrm{d}, *}$ \\ ${ }^{a}$ Dipartimento C.I.M.A., Istituto ISTM, Via Venezian 21, Milano 20133, Italy \\ ${ }^{\mathrm{b}}$ Dipartimento di Biotecnologie e Bioscienze, Università di Milano-Bicocca, Piazza della Scienza 2, Milano 20100, Italy \\ ${ }^{\mathrm{c}}$ Istituto Sperimentale per la Nutrizione delle Piante, Via Pianezza 115, Torino 10151, Italy \\ d Dipartimento di Chimica Generale, Università di Pavia, Via Taramelli 12, Pavia 27100, Italy
}

Received 29 July 2003; received in revised form 26 September 2003; accepted 6 October 2003

\begin{abstract}
The present investigation addresses the problem of the binding mode of phenolic inhibitors and the substrate ascorbate to the active site of ascorbate oxidase. The results from both types of compounds indicate that the binding site is located in a pocket near the type 1 copper center. This information is of general interst for blue multicopper oxidases. Docking calculations performed on the ascorbate oxidase-ascorbate complex show that binding of the substrate occurs in a pocket near type $1 \mathrm{Cu}$, and is stabilized by at least five hydrogen bonding interactions with protein residues, one of which involves the His512 $\mathrm{Cu}$ ligand. Similar docking studies show that the isomeric fluorophenols, which act as competitive inhibitors toward ascorbate, bind to the enzyme in a manner similar to ascorbate. The docking calculations are supported by ${ }^{19} \mathrm{~F}$ NMR relaxation measurements performed on fluorophenols in the presence of the enzyme, which show that the bound inhibitors undergo enhanced relaxation by the paramagnetic effect of a nearby $\mathrm{Cu}$ center. Unambiguous support to the location of the inhibitor close to type $1 \mathrm{Cu}$ was obtained by comparative relaxation measurements of the fluorophenols in the presence of the ascorbate oxidase derivative where a $\mathrm{Zn}$ atom selectively replaces the paramagnetic type $2 \mathrm{Cu}$. The latter experiments show that contribution to relaxation of the bound inhibitors by the type $2 \mathrm{Cu}$ site is negligible.
\end{abstract}

(c) 2003 Elsevier Ltd. All rights reserved.

Keywords: Ascorbate oxidase; Fluorophenols; Enzyme inhibition; NMR relaxation; Docking calculations

Abbreviations: AO, ascorbate oxidase; T2D-AO, type 2 copper depleted derivative of AO; ZnT2-AO, AO derivative with type 2 copper substituted by zinc; $\mathrm{Zn}_{4}-\mathrm{AO}$, $\mathrm{AO}$ derivative with all copper ions substituted by zinc; DDC, diethyl dithiocarbamate; EDTA, ethylenediaminetetraacetic acid; EPR, electron paramagnetic resonance; NMR, nuclear magnetic resonance; $\mathrm{CD}$, circular dichroism; MM, molecular mechanics; SA, simulated annealing

* Corresponding author. Tel.: +39-0382-507331; fax: +39-0382-528544.

E-mail address: bioinorg@unipv.it (L. Casella).

\section{Introduction}

Ascorbate oxidase (AO) is a dimeric coppercontaining enzyme which catalyzes the four-electron reduction of dioxygen to water using preferably $\mathrm{L}$-ascorbate as a substrate. The enzyme belongs to the family of multicopper blue oxidases which includes laccases, ceruloplasmin, and other less well characterized enzymes (Solomon, Sundaram, \& Machonkin, 

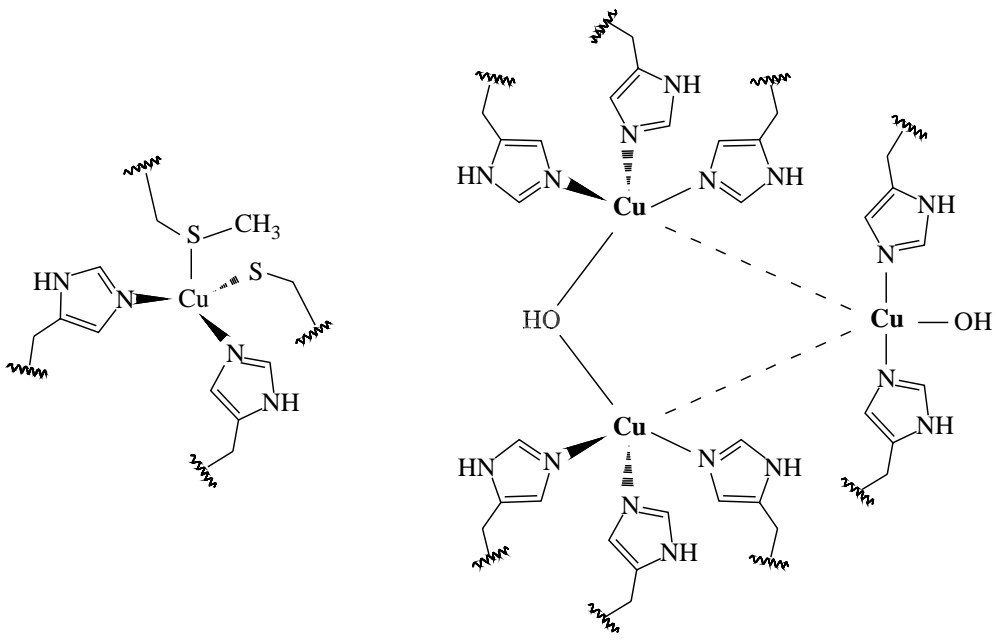

Scheme 1.

1996). These oxidases contain four copper atoms per functional unit, which are classified as types 1 , 2 , and 3 according to their spectroscopic and redox properties (Reinhammar \& Malmström, 1981). Ascorbate oxidase is the first of the blue oxidases to be structurally characterized (Messerschmidt, Rossi, Ladenstein, Huber, Bolognesi, Gatti, Marchesini, Petruzzelli, \& Finazzi-Agrò, 1989; Messerschmidt, Ladenstein, Huber, Bolognesi, Avigliano, Petruzzelli, Rossi, \& Finazzi-Agrò, 1992). X-ray crystallographic information is now available for a number of $\mathrm{AO}$ derivatives (Messerschmidt, Luecke, \& Huber, 1993; Messerschmidt, 1997). The active site contains the mononuclear type 1 copper separated by about $12.5 \AA$ from the trinuclear cluster comprising the types 2 and 3 coppers (Scheme 1). The type 1 copper contains the canonical two His, Met and Cys ligand set. The pair of type 3 copper atoms has a total of six histidine ligands (three His per $\mathrm{Cu}$ ) in a trigonal prismatic arrangement and is bridged by an $\mathrm{OH}^{-}\left(\right.$or $\left.\mathrm{O}^{2-}\right)$ group, which strongly couples the two metal ions and makes this dinuclear center EPR silent. The type 2 copper has two histidine ligands and an $\mathrm{OH}^{-}\left(\right.$or $\left.\mathrm{H}_{2} \mathrm{O}\right)$ ligand in a trigonal arrangement. The three $\mathrm{Cu}$ atoms of the cluster are all close to each other, with an average $\mathrm{Cu}-\mathrm{Cu}$ distance of $3.7 \AA$ and individual distances within $0.16 \AA$ from this value (Messerschmidt, 1997). These active site structural features have been subsequently found, basically unchanged, in the X-ray crystallographic determination of human ceruloplas- min (Zaitseva, Zaitsev, Card, Moshkov, Box, Ralph, \& Lindley, 1996; Lindley, Zaitseva, Zaitsev, Card, Moshkov, \& Box, 1997) which, on the other hand, contains two additional type 1 copper centers, and fungal laccase (Bertrand, Jolivat, Briozzo, Caminade, Joly, Madzak, \& Mougin, 2002; Hakulinen, Kiiskinen, Kruus, Saloheimo, Paananen, Koivula, \& Rouvinen, 2002). With the latter enzyme, recent spectroscopic studies suggested that multiple forms of the trinuclear cluster may be present, since the type of magnetic coupling between the coppers seems to vary with conditions (Fraterrigo, Miller, Reinhammar, \& McMillin, 1999; Li, McMillin, \& Antholine, 1992; Huang, Sakurai, Maritano, Marchesini, \& Suzuki, 1999; Gromov, Marchesini, Farver, Pecht, \& Goldfarb, 1999).

During activity, the blue copper oxidases couple the four-electron reduction of dioxygen to water with one-electron oxidation of four molecules of the substrate. For AO, the fastest and possibly physiological substrate is L-ascorbate (Kroneck, Armstrong, Merkle, \& Marchesini, 1982; Avigliano \& Finazzo-Agrò, 1997), which in the common plant sources of the enzyme is involved in cell processes associated with plant growth, protection and development (Arrigoni, 1994; Cordoba \& Gonzales-Reyes, 1994; Wheeler, Jones, \& Smirnoff, 1998). The complex mechanism through which the enzyme catalysis proceeds is far from being understood (Solomon et al., 1996; Zaitseva et al., 1996; Casella, Monzani, Santagostini, 
De Gioia, Gullotti, Fantucci, Beringhelli, \& Marchesini, 1999). An essential aspect of the catalytic mechanism is the mode of interaction and reaction of the organic substrate, ascorbate in the case of AO, with the enzyme. This problem has received limited attention so far, and only recently the X-ray structure of a fungal laccase showed that binding of an arylamine occurs near type 1 copper (Bertrand et al., 2002). In the case of $\mathrm{AO}$, the problem of substrate binding has been so far approached through kinetic and inhibition studies (Casella et al., 1999; Wimalasena \& Dharmasena, 1994; Marchesini, Cappelletti, Canonica, Danieli, \& Tollari, 1977; Gaspard, Monzani, Casella, Gullotti, Maritano, \& Marchesini, 1997). In this paper, we gain a better definition of the ascorbate binding site in AO by reporting kinetic and ${ }^{19} \mathrm{~F}$ NMR relaxation studies, and docking calculations on the competitive inhibitory interaction of fluorophenols with the enzyme. The presence of a fluorine atom in these molecules bears the advantage to allow studying relaxation effects in the same aqueous buffer solution used in the inhibition studies. In order to assess the contribution of individual paramagnetic centers of AO to the relaxation of bound inhibitor nuclei, the zinc containing protein derivatives $\mathrm{ZnT} 2-\mathrm{AO}$ and $\mathrm{Zn}_{4}-\mathrm{AO}$ have been also studied.

\section{Materials and methods}

Ascorbate oxidase was isolated and purified from green zucchini squash (Cucurbita pepo) according to the procedure of Marchesini and Kroneck (1979). The protein had optical indices $A_{280} / A_{610}=24.4$ and $A_{330} / A_{610}=0.8$ in $0.1 \mathrm{M}$ phosphate buffer, pH 5.7. The enzyme was routinely conserved as microcrystals in ammonium sulphate. Solutions of the enzyme were obtained after dialysis of the crystals against the appropriate buffer. Protein concentrations were determined spectrophotometrically assuming $M_{\mathrm{r}}=$ 140,000 and $\varepsilon_{610}=9700 \mathrm{M}^{-1} \mathrm{~cm}^{-1}$ for the dimeric enzyme. Buffer solutions were prepared with water purified in a Millipore Milli-Q system.

UV-Vis spectra were recorded on a diode array HP 8452A spectrophotometer equipped with a thermostated cell holder maintained at $25 \pm 0.1^{\circ} \mathrm{C}$. Enzyme activity and inhibition kinetics of ascorbate oxidation were determined by measuring dioxygen consumption on an Amel 360 oxygen meter fitted with a Clark electrode. The activity is expressed in Dawson units, where 1 Dawson unit $=1 \mu \mathrm{mol} \mathrm{m^{-1 }}$ ascorbate oxidized (mg enzyme $)^{-1}$. The present enzyme preparation exhibited very high activity, above 3900 Dawson units in 0.1 M phosphate buffer, pH 5.7. EDTA $(1 \mathrm{mg} / \mathrm{ml})$ was added to stabilize ascorbic acid solutions in the buffer. The concentration of ascorbic acid was determined from its absorbance at $266 \mathrm{~nm}$ using $\varepsilon_{266}=15,000 \mathrm{M}^{-1} \mathrm{~cm}^{-1}$.

The ZnT2-AO derivative was prepared by addition of DDC to the native enzyme (in a molar ratio of 10:1); the blue enzyme solution turned green upon addition of the reagent. After incubation for about $30 \mathrm{~min}$ at $4{ }^{\circ} \mathrm{C}$, the brown precipitate was eliminated by centrifugation (about $90 \mathrm{~min}$ at $18,700 \times \mathrm{g}$ ). The enzyme solution was then dialysed for $4 \mathrm{~h}$ against $0.1 \mathrm{M}$ phosphate buffer at $\mathrm{pH} 5.7$ using a collodion bag, in order to eliminate traces of the $\mathrm{Cu}$-DDC complex previously formed. Then, $\mathrm{ZnSO}_{4}$ was added for three times to the enzyme solution, with a molar ratio of 20:1 to the protein each time. After each addition, the solution was left at $4{ }^{\circ} \mathrm{C}$ for $15 \mathrm{~min}$ and then dialyzed against $0.1 \mathrm{M}$ phosphate buffer $\mathrm{pH}$ 5.7. Zinc analysis by atomic absorption gave $2.2 \pm 0.2$ atoms per protein dimer.

The $\mathrm{Zn}_{4}$-AO derivative was prepared by dialysing anaerobically the native enzyme for three times $(2 \mathrm{~h}$ each time) against a degassed $0.1 \mathrm{M}$ phosphate buffer solution at $\mathrm{pH} 7.0$ containing $\mathrm{KCN}(25 \mathrm{mM})$ and a small amount of ascorbic acid, in order to eliminate traces of dissolved oxygen in the solution. The resulting colorless apoenzyme solution was dialysed three times ( $2 \mathrm{~h}$ each time) against $0.1 \mathrm{M}$ phosphate buffer pH 5.7 containing about $1 \mathrm{mg} / \mathrm{ml}$ EDTA, in order to remove traces of copper and other ions, and three times against the same buffer solution without EDTA. Then, $\mathrm{ZnSO}_{4} \cdot 7 \mathrm{H}_{2} \mathrm{O}$ was added for three times to the apoenzyme solution, with a molar ratio of 20:1 each time. After each addition, the enzyme solution was left at $4{ }^{\circ} \mathrm{C}$ for $15 \mathrm{~min}$ and then dialysed against $0.1 \mathrm{M}$ phosphate buffer $\mathrm{pH}$ 5.7. The residual amount of enzyme containing type $1 \mathrm{Cu}$ was about $10 \%$ as estimated from the $A_{280} / A_{610}$ ratio in the UV-Vis spectrum. Zinc analysis of this protein derivative by atomic absorption gave $7.3 \pm 0.5$ atoms per protein dimer.

Inhibition studies were performed with the three isomeric fluorophenols in $0.1 \mathrm{M}$ phosphate buffer $\mathrm{pH}$ 5.7 , by measuring the activity as a function of ascorbic 
acid concentration. Typically, the experiments were carried out with inhibitor concentration ranging from 0 and $50 \mathrm{mM}$ and ascorbic acid concentration varying from 0 to $3 \mathrm{mM}$. The enzyme concentration was about $3 \mathrm{nM}$. The reaction rate was measured from the initial slope of the dioxygen consumption curve. The kinetic and inhibition parameters were obtained from fitting of the plots of initial rate versus initial ascorbic acid concentration, along with the appropriate replots (Segel, 1975).

Relaxation measurements were performed on a $400 \mathrm{MHz}$ Bruker AVANCE NMR spectrometer at $25 \pm 0.1^{\circ} \mathrm{C}$ with a $50 \mathrm{mM}$ solution of inhibitor in $0.1 \mathrm{M}$ phosphate buffer, $\mathrm{pH} 5.7$, containing $50 \% \mathrm{D}_{2} \mathrm{O}$. Relaxation time $T_{1}$ was measured for the fluorine nucleus of the inhibitor at different enzyme concentrations, ranging from 0 to $10^{-5} \mathrm{M}$, in order to obtain information about distance of fluorine from copper ions contained in the active site of AO. The same experiments were performed using $\mathrm{ZnT} 2-\mathrm{AO}$ and $\mathrm{Zn}_{4}-\mathrm{AO}$ instead of native $\mathrm{AO}$.

Longitudinal relaxation rates were determined using the standard inversion recovery method (Vold, Waugh, Klein, \& Phelps, 1968). From the values of $T_{1 \mathrm{obs}}$ it is possible to calculate $T_{1 \mathrm{~b}}$, which is the $T_{1}$ for the bound inhibitor when this is in fast exchange between the active site and the bulk solution, from the equation:

$\frac{1}{T_{1 \mathrm{obs}}}=\left(\frac{1}{T_{1 \mathrm{~b}}}-\frac{1}{T_{1 \mathrm{f}}}\right) \frac{\left[E_{0}\right]}{K_{\mathrm{I}}+\left[I_{0}\right]}+\frac{1}{T_{1 \mathrm{f}}}$

where $T_{1 \mathrm{f}}$ is $T_{1}$ for the free inhibitor, $\left[E_{0}\right]$ and $\left[I_{0}\right]$ are the initial enzyme and inhibitor concentrations, respectively, and $K_{\mathrm{I}}$ is the dissociation constant for the enzyme-inhibitor complex. $T_{1 \mathrm{~b}}$ is related to the paramagnetic contribution to the relaxation, $T_{1 \mathrm{M}}$, through the equation:

$\frac{1}{T_{1 \mathrm{~b}}}=\frac{1}{T_{1 \mathrm{D}}}+\frac{1}{T_{1 \mathrm{M}}+\tau_{\mathrm{M}}}$

where $\tau_{\mathrm{M}}$ is the lifetime for chemical exchange and $T_{1 \mathrm{D}}$ corresponds to the diamagnetic contribution to the relaxation for the bound inhibitor. In the presence of strong paramagnetic effect, the diamagnetic contribution can be neglected, and assuming that the exchange between the free and bound state is fast, Eq. (2) yields $T_{1 \mathrm{~b}}=T_{1 \mathrm{M}}$.
The paramagnetic contribution to the relaxation rate, $\left(T_{1 \mathrm{M}}\right)^{-1}$, of the fluorine nucleus of the bound inhibitor arising from the unpaired electron on the copper center(s) is related to the copper-fluorine distance $(r)$ by the Solomon-Bloemberger equation (Solomon \& Bloembergen, 1956; Bertini \& Luchinat, 1996). In the present case, since the inhibitor is not bound to the metal center(s), only the dipolar term is retained in this equation, while the scalar term can be neglected (Bertini \& Luchinat, 1996).

$$
\begin{aligned}
\frac{1}{T_{1 \mathrm{M}}}= & \frac{2}{15}\left(\frac{\mu_{0}}{4 \pi}\right)^{2} \frac{\gamma_{\mathrm{I}}^{2} g^{2} \mu_{\mathrm{B}}^{2} S(S+1)}{r^{6}} \\
& \times\left(\frac{3 \tau_{\mathrm{C}}}{1+\omega_{\mathrm{I}}^{2} \tau_{\mathrm{C}}^{2}}+\frac{7 \tau_{\mathrm{C}}}{1+\omega_{\mathrm{S}}^{2} \tau_{\mathrm{C}}^{2}}\right)
\end{aligned}
$$

where $\mu_{0}$ is the permeability of vacuum, $\omega_{\mathrm{I}}$ and $\omega_{\mathrm{S}}$ are nuclear and electronic Larmor angular velocities, respectively, $\gamma_{\mathrm{I}}$ is the gyromagnetic ratio of the nucleus, $g$ the electron isotropic $g$-factor, $\mu_{\mathrm{B}}$ the Bohr magneton, $S$ the spin quantum number of the electron, and $\tau_{\mathrm{C}}$ the effective correlation time of the dipolar interaction. In spite of the assumptions underlying the use of this equation, it proved to be useful in our previous studies on peroxidases (Casella, Poli, Gullotti, Selvaggini, Beringhelli, \& Marchesini, 1994; Redaelli, Monzani, Santagostini, Casella, Sanangelantoni, Pierattelli, \& Banci, 2002) and ascorbate oxidase (Gaspard et al., 1997). In the case of copper enzymes, it is generally assumed that the overall correlation time is determined by the electronic relaxation, and a $\tau_{\mathrm{C}}$ value of $3.5 \times 10^{-9} \mathrm{~s}$ has been estimated for a variety of copper enzymes (Bertini, Briganti, Luchinat, Mancini, \& Spina, 1985; Williams \& Falk, 1986). The same $\tau_{C}$ value has been employed here.

$\mathrm{CD}$ spectra of $\mathrm{AO}$ derivatives were recorded on a JASCO J-500 spectropolarimeter in the range $300-700 \mathrm{~nm}$. The experiments were carried out using $o$ - and $p$-fluorophenol as inhibitors, with a concentration varying from 0 to $30 \mathrm{mM}$. The enzyme concentration was about $10^{-5} \mathrm{M}$.

\subsection{Docking simulations}

The docking algorithm used in the present investigation, which is an improved version of a previously reported procedure developed to study enzyme-substrate interactions (Casella et al., 1999), 
is based on a combined use of random search and total energy minimization including the relevant degrees of freedom of the composite system. In particular, the algorithm is a multi-step optimization, each step being composed by two parts: the first, of SA type, is carried out only on the subspace of the mutual roto-translations, and produces a global minimum of the intermolecular energy when the two molecules are considered as rigid bodies. The second part is a conventional, gradient based, geometry optimization carried out within the MM approximations and uses as starting point the minimum reached by the SA step. The MM calculation allows a complete relaxation of the internal degrees of freedom of the interacting molecules, thus keeping into account subtle effects such as the slight movement of some amino acid side chains to better accommodate the docking partner. The SA-MM steps are repeated in cycles, until the MM total energy of the guest-host composite system is converged. This combined algorithm has been implemented in a new computer program (P. Fantucci, L. De Gioia, E. Virzi, to be submitted), automatically interfaced to the GROMOS96 suite of programs (Van Gunsteren \& Berendsen, 1990), which provides the MM part of the combined procedure, as well as the general dictionary for fragments and forcefield parameters.
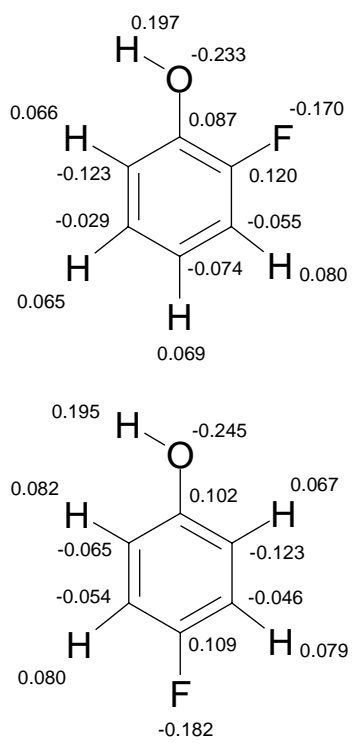

The interaction (docking) energy was calculated as:

$E_{\text {docking }}=E_{\mathrm{p}-\mathrm{s}}-E_{\mathrm{p}}-E_{\mathrm{s}}$

where $E_{\mathrm{p}-\mathrm{s}}$ is the energy of the protein ligand adduct obtained from the SA/MM docking optimization, $E_{\mathrm{p}}$ the energy of the isolated protein after MM optimization, and $E_{\mathrm{s}}$ the energy of the isolated organic ligand after MM optimization.

The structural and electronic parameters of all the small organic molecules used in this study were calculated using the semiempirical method AM1 (Dewar, Zoebisch, Healy, \& Stewart, 1985) and transferred to the GROMOS96 forcefield. The monoanionic form of ascorbate was used in the calculations because this is the most stable form present in physiological conditions, whereas fluorophenols were described as neutral molecules. Fig. 1 shows the structures of the organic ligands used in this study and their associated partial atomic charge values, as calculated by AM1 optimization. Note that, even if the total -1 charge of ascorbate can be formally localized on one of the three exocyclic oxygen atoms, AM1 calculations result in an almost even distribution of the electron density on the three atoms, irrespective of the presence of the proton on the central one. Moreover, the conformational properties of ascorbate have been investigated sampling the conformational space produced by the
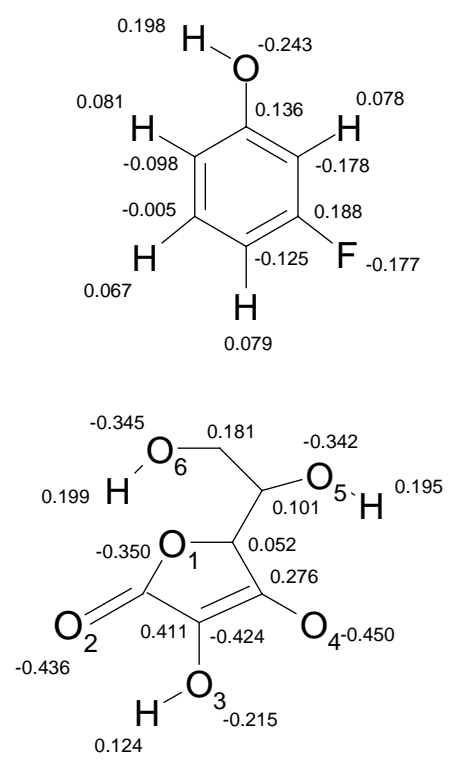

Fig. 1. Structure and partial atomic charges, as obtained by AM1 optimization, of the organic ligands investigated in the present study. 


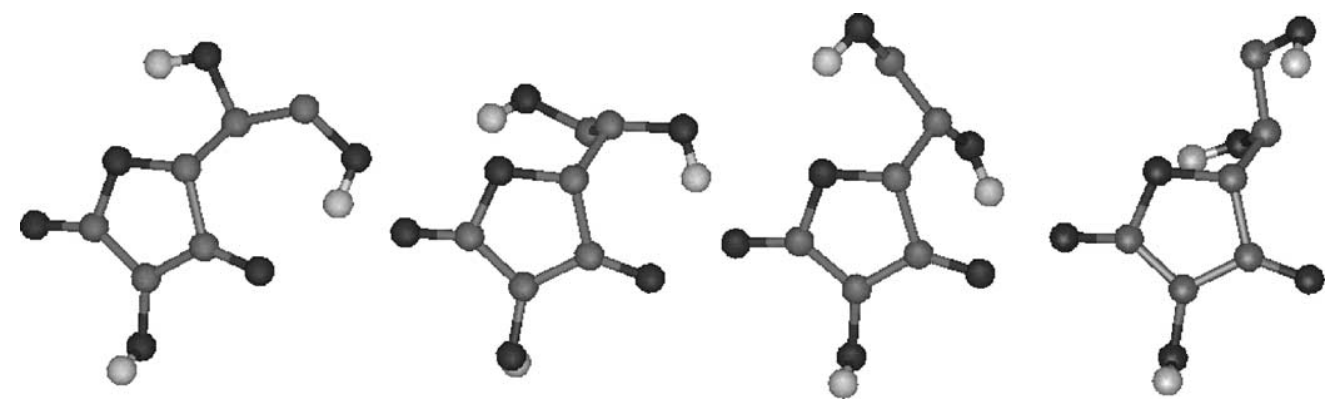

Fig. 2. Low energy conformers of ascorbate, as obtained by sampling of the conformational space of ascorbate by AM1 optimization.

discrete rotation (steps of $20^{\circ}$ ) along the two $\mathrm{C}-\mathrm{C}$ bonds characterizing the diol side chain. The AM1 optimization of the generated structures converged to four low energy conformers, differing by no more than $5 \mathrm{kcal} \mathrm{mol}^{-1}$, that were used as starting points in the docking procedure (see Fig. 2). Note also that the partial atomic charge values computed for the different conformers of L-ascorbate differ by less than 0.02 a.u. (data not shown).

\section{Results}

\subsection{Kinetics}

Like the phenolic compounds studied previously (Gaspard et al., 1997), the three isomeric fluorophenols inhibit the ascorbic acid oxidation catalyzed by AO. To determine the type of inhibition, the enzymatic reaction was studied over a range of ascorbic acid concentrations in the presence of several fixed amounts of the inhibitor. For $p$-fluorophenol and $m$-fluorophenol the inhibition was found to be competitive, as for the phenolic compounds studied before. With $o$-fluorophenol, the double reciprocal plots of initial rates at different inhibitor concentration $v s$. ascorbic acid concentration were found to intersect on the left of the $y$-axis, indicating mixed type inhibition (Segel, 1975). Analysis of the kinetic data gave the inhibition constants for the fluorophenols isomers; for para $K_{\mathrm{I}}=15 \pm 4 \mathrm{mM}$, meta $K_{\mathrm{I}}=80 \pm 6 \mathrm{mM}$, and ortho $K_{\mathrm{I}}=36 \pm 7 \mathrm{mM}$ and $K_{\mathrm{I}}^{\prime}=116 \pm 54 \mathrm{mM}$, where the constants correspond to the following association equilibria:

$$
K_{\mathrm{I}}: \quad \mathrm{E}+\mathrm{I} \rightleftharpoons \mathrm{EI}
$$

$K_{\mathrm{I}}^{\prime}: \quad \mathrm{ES}+\mathrm{I} \rightleftharpoons \mathrm{ESI}$

where E: enzyme, I: inhibitor, and S: substrate. Therefore, even though $o$-fluorophenol binds both to the enzyme and the enzyme-substrate complex, the former binding is stronger and is mostly responsible for the inhibition effect.

\subsection{Characterization of ZnT2-AO and Zn4-AO}

Replacement of type $2 \mathrm{Cu}$ by $\mathrm{Zn}$ leads to the enzymatically inactive ZnT2-AO derivative. As expected, the type $2 \mathrm{Cu}$ features are absent in the EPR spectrum of this derivative, which is thus the same as that we reported for T2D-AO (Casella, Gullotti, Pallanza, Pintar, \& Marchesini, 1991), while the optical and CD spectra of the enzyme in the near UV-Vis range are little affected by the metal replacement, since they are dominated by the type $1 \mathrm{Cu}$ characteristics. The only appreciable change occurs in the $\mathrm{CD}$ range between 300 and $350 \mathrm{~nm}$, where the weak CD activity of negative sign of the native protein is significantly reduced in ZnT2-AO (data not shown). This effect is due both to type 2 copper depletion, since this center also contributes to the absorption intensity in the $300-350 \mathrm{~nm}$ range (Casella et al., 1991), and to the fact that a portion of type 3 copper may remain in the reduced state in ZnT2-AO, as it is in T2D-AO (Casella et al., 1991) and in T2D-laccase (Kau, Spira-Solomon, Penner-Hahn, Hodgson, \& Solomon, 1987).

For $\mathrm{Zn}_{4}$-AO the spectral characterization is necessarily limited by the absence of suitable chromophores. The CD spectrum of this derivative in the aromatic region exhibits less well resolved features of positive sign at 283, 291 and $296 \mathrm{~nm}$, attributed 
to tyrosine and tryptophan residues, compared to AO (Casella, Fantucci, Gullotti, \& Marchesini, 1984) and ZnT2-AO. This is indicative of some local conformational rearrangement occurring on metal replacement.

\subsection{NMR relaxation measurements}

The paramagnetic effect of types 1 and 2 copper ions contained in the enzyme active site on the longitudinal relaxation of ${ }^{19} \mathrm{~F}$ nuclei of the bound fluorophenols could give information about $\mathrm{Cu}-\mathrm{F}$ distances in the enzyme-inhibitor complexes. To calculate these distances, $T_{1}$ was determined for the fluorophenol alone and in presence of variable amounts of $\mathrm{AO}, \mathrm{ZnT} 2-\mathrm{AO}$ or $\mathrm{Zn}_{4}-\mathrm{AO}$. For the native protein, paramagnetic contributions to nuclear relaxation can be expected by types 1 and 2 coppers, whereas type 3 copper is strongly coupled and its contribution can be neglected. Therefore, the $\mathrm{Cu}-\mathrm{F}$ distances calculated for ZnT2-AO-fluorophenol complexes represent distances of the bound inhibitor nuclei from type 1 copper. The experiments with $\mathrm{Zn}_{4}-\mathrm{AO}$ were needed to assess the $1 / T_{1 \mathrm{D}}$ contribution to the relaxation rate. On binding to the large protein molecule, the tumbling motion of the fluorophenols becomes slower and this effect must be taken into account in the determination of $1 / T_{1 \mathrm{~b}}$. Upon subtracting the (small) contribution to relaxation rate due to binding to the diamagnetic protein, it was possible to get an estimate of the $\mathrm{Cu}-\mathrm{F}$ distances for the protein complexes with $o$ - and $p$-fluorophenol from the calculated $1 / T_{1 \mathrm{~b}}$ values, as collected in Table 1 . We could not perform reliable measurements of the $T_{1 \mathrm{~b}}$ values for $m$-fluorophenol in the presence of AO or ZnT2-AO because progressive additions of the proteins to the inhibitor solutions in the NMR tube invariably caused formation of protein precipitates at some stages.

\section{4. $C D$ and EPR experiments on $A O-$ phenol and ZnT2-AO-phenol complexes}

The CD and EPR spectra of AO and ZnT2-AO in the presence of the fluorophenols were studied at moderate concentrations of the inhibitors (up to $50 \mathrm{mM}$ ) since with larger concentrations of these compounds some denaturation of the proteins occurred. In any case, neither types of spectra were appreciably affected by the presence of the inhibitors.

\subsection{Docking calculations}

The computational investigation of AO was carried out with the aim of describing which amino acids forming the active site are involved in molecular recognition and/or electron transfer when organic molecules such as L-ascorbate (substrate) or fluorophenols (inhibitors) interact with the enzyme. The computational docking study carried out as described under Section 2 and using L-ascorbate as binding donor shows that His512, one of the ligands of type 1 copper, interacts directly with the substrate and is possibly involved in the electron transfer process, whereas His445 and the other copper ligands are not involved in the recognition event. A number of interactions between the enzyme active site and L-ascorbate were observed (Fig. 3): the NH group of His512 forms a hydrogen bond with the $\mathrm{O}_{3}$ atom of ascorbate (atoms labeled according to Fig. 1); the amidic group of Gln353 is hydrogen bonded to the hydroxyl group $\mathrm{O}_{5}-\mathrm{H}$ of ascorbate; the indole $\mathrm{NH}$ of $\operatorname{Trp} 362$ forms an $\mathrm{H}$-bond with $\mathrm{O}_{2}$; the carboxylate of G1u443 interacts with $\mathrm{O}_{6}-\mathrm{H}$; the aromatic ring of Trp163 is involved in a stacking interaction with the lactone ring of $\mathrm{L}$-ascorbate and the side chain of $\operatorname{Arg} 285$ forms hydrogen bonds with $\mathrm{O}_{4}$. This complex between AO and L-ascorbate is characterized by a favorable docking energy and is more stable by $5 \mathrm{kcal} \mathrm{mol}^{-1}$ with respect to a second complex

Table 1

Longitudinal relaxation time for the fluorine nuclei of the bound inhibitors and copper-fluorine distances evaluated with Eq. (3)

\begin{tabular}{|c|c|c|c|c|c|}
\hline \multirow[t]{2}{*}{ Inhibitor } & \multirow[t]{2}{*}{$\delta F(\mathrm{ppm})^{\mathrm{a}}$} & \multicolumn{2}{|l|}{$\mathrm{AO}$} & \multicolumn{2}{|l|}{ ZnT2-AO } \\
\hline & & $T_{1 \mathrm{~b}}(\mathrm{~ms})$ & $r(\mathrm{Cu}-\mathrm{F})(\AA)$ & $T_{1 \mathrm{~b}}(\mathrm{~ms})$ & $r(\mathrm{Cu}-\mathrm{F})(\AA)$ \\
\hline$p$-Fluorophenol & -126.0 & 3.3 & 5.9 & 3.7 & 6.0 \\
\hline$o$-Fluorophenol & -138.2 & 2.0 & 5.4 & 2.2 & 5.5 \\
\hline
\end{tabular}

${ }^{\text {a }}$ Fluorine chemical shifts are relative to $\mathrm{CFCl}_{3}$. 

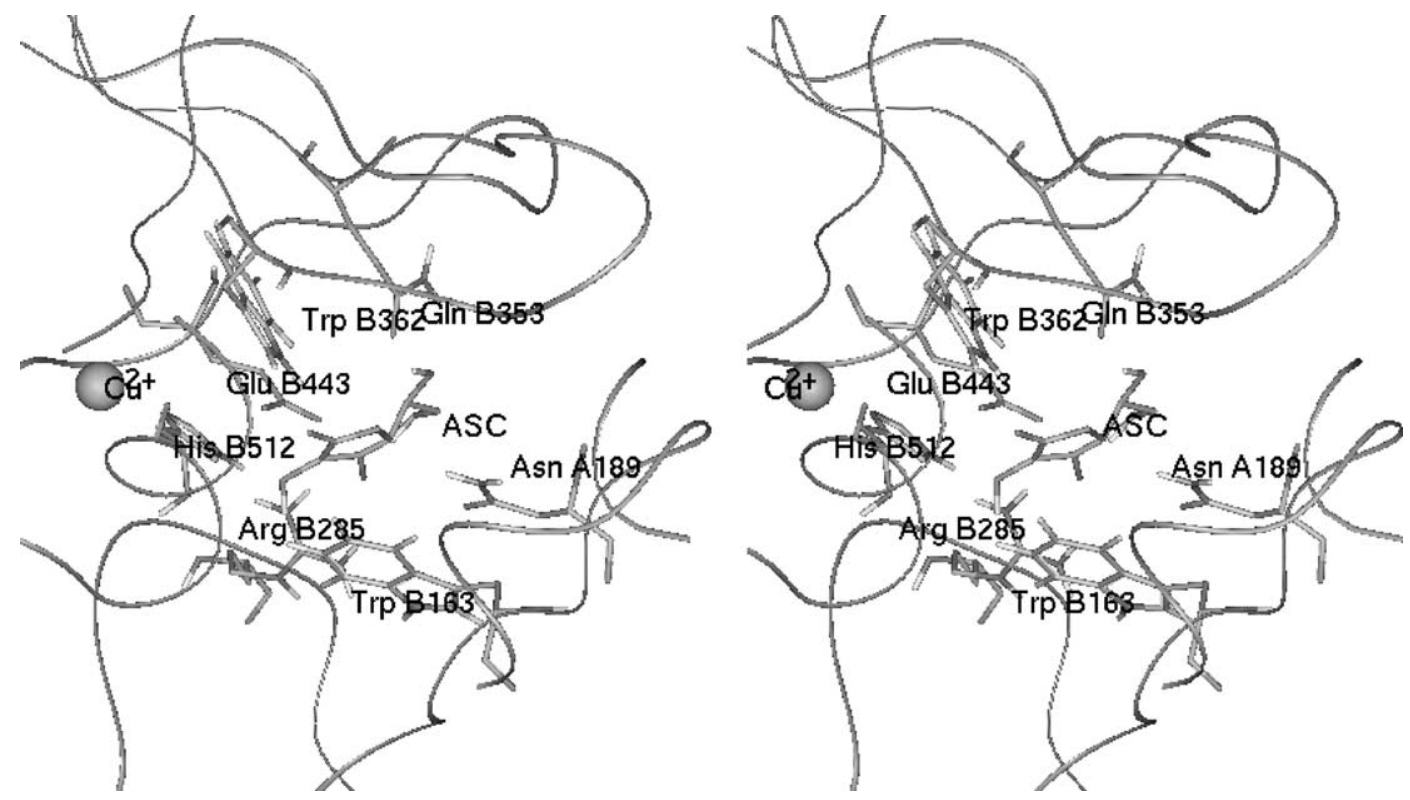

Fig. 3. Lowest energy minimum structure of the AO-ascorbate complex as obtained by computational docking (stereo view). For the sake of clarity only the side chains of the amino acids involved in ascorbate (ASC) recognition and the copper center have been explicitly shown.
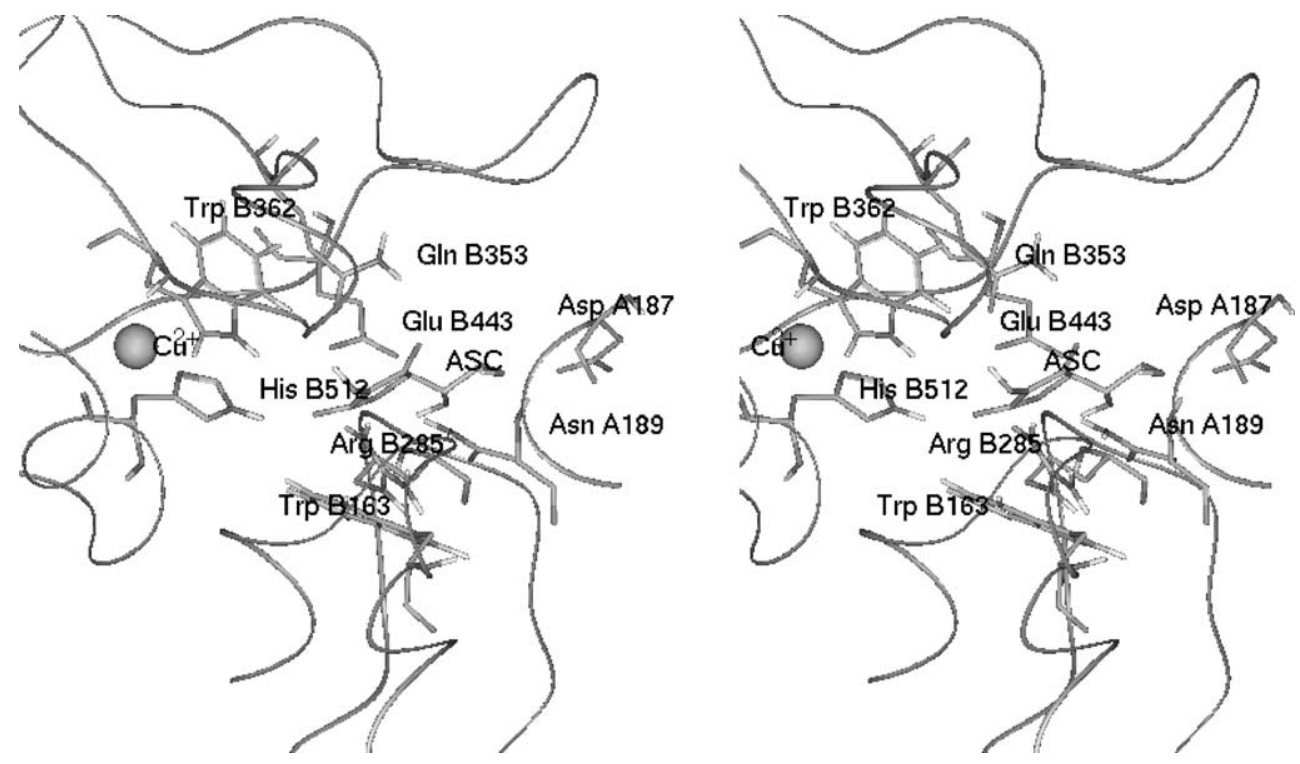

Fig. 4. Low energy minimum structure (the total energy is $5 \mathrm{kcal} \mathrm{mol}^{-1}$ higher with respect to the adduct shown in Fig. 1) of the AO-ascorbate complex as obtained by computational docking (stereo view). For the sake of clarity only the side chains of the amino acids involved in ascorbate (ASC) recognition and the copper center have been explicitly shown. 


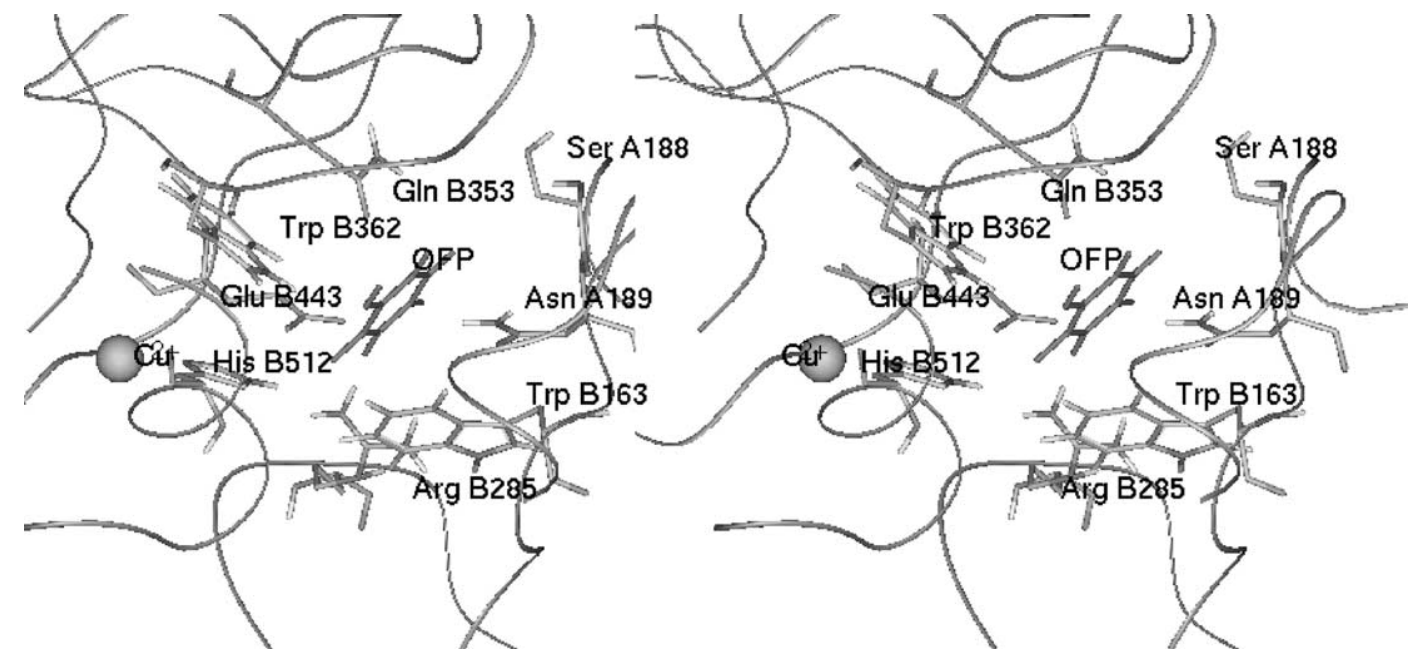

Fig. 5. Lowest energy minimum structure of the adduct between AO and $o$-fluorophenol (OFF) as obtained by computational docking (stereo view). For the sake of clarity only the side chains of the amino acids involved in phenol recognition and the copper center have been explicitly shown.

located by the GSA/MM procedure where the residues involved in the L-ascorbate recognition are the same as those previously described but the orientation of the substrate in the enzyme active site is slightly different (Fig. 4). In fact, in the latter complex, it is the $\mathrm{O}_{2}$ atom of L-ascorbate which is involved in hydro- gen bonding with His512. In addition, the substrate forms hydrogen bonds also with Gln353 and Arg285.

The computational results obtained for AO-inhibitor complexes through the combined GSA/MM methods are in agreement with the kinetic data, since the fluorophenols fit to the AO binding site. The three
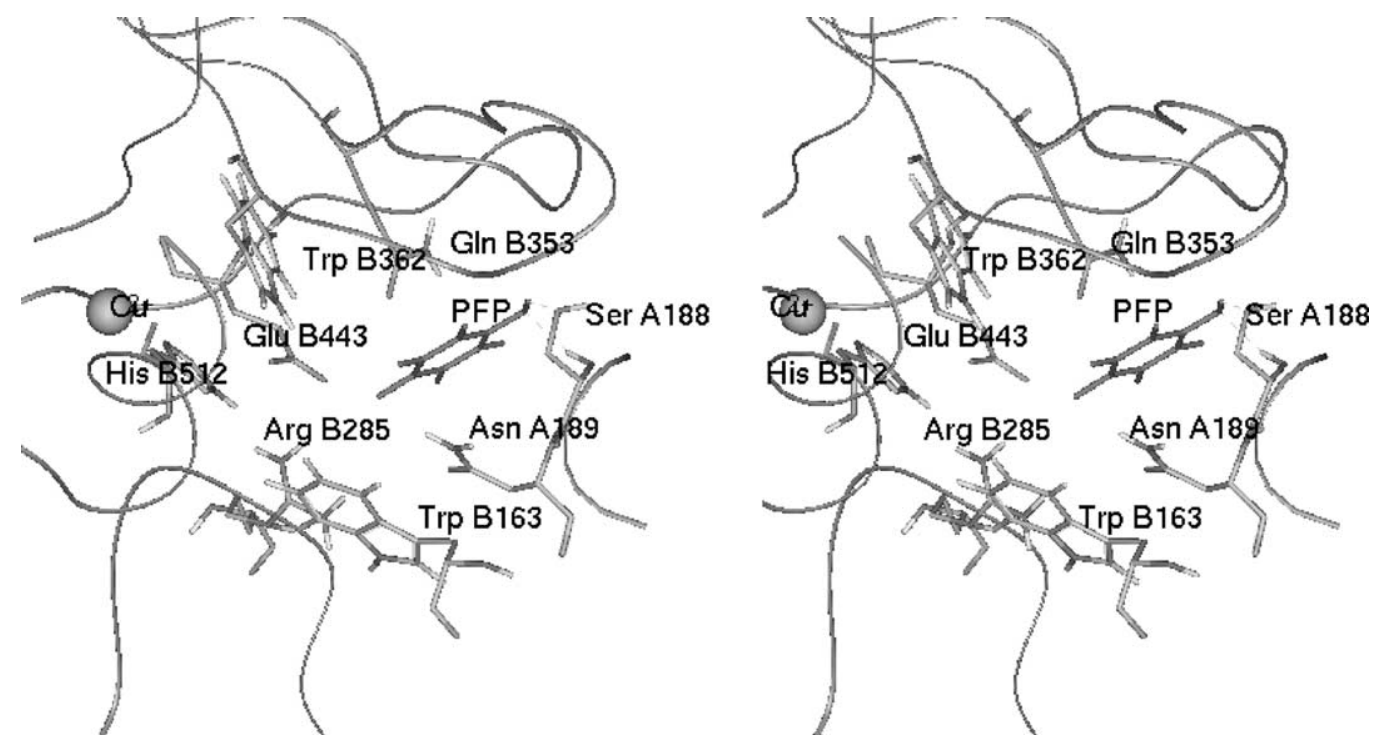

Fig. 6. Lowest energy minimum structure of the adduct between $\mathrm{AO}$ and $p$-fluorophenol (PFF) as obtained by computational docking (stereo view). For the sake of clarity only the side chains of the amino acids involved in phenol recognition and the copper center have been explicitly shown. 
AO-fluorophenol complexes obtained sampling the whole crevice forming the active site and featuring the lowest intermolecular energy are characterized by a phenol disposition in the active site which is very similar to that observed for L-ascorbate. This indicates that the presence of the inhibitor does not allow proper recognition of L-ascorbate by the enzyme. In particular, $m$ - and $o$-fluorophenol are characterized by a very similar spatial disposition of the aromatic ring and by the presence of a hydrogen bond between His512A and the fluorine atom (Fig. 5), whereas $p$-fluorophenol adopts a slightly different orientation due to an $\mathrm{H}$-bond formed between the $\mathrm{OH}$ group and the Serl88B residue that is located on the other $\mathrm{AO}$ monomer forming the dimer (Fig. 6). Note that Trpl63B plays a crucial role in all cases, being involved in a stacking interaction with the aromatic ring of the inhibitors. These spatial arrangements result in $\mathrm{Cu}-\mathrm{F}$ distances equal to $6.80,6.96$ and $9.11 \AA$ for $o-$, $m$ - and $p$-fluorophenol, respectively. It is worth noting that the substituents on the phenyl ring in $o$ - and $m$-fluorophenol are both deeply buried in the active site, resulting in some unfavorable steric interactions. On the contrary, $p$-fluorophenol is less buried resulting in a less strained arrangement of the amino acids forming the active site.

\section{Discussion}

Ascorbate oxidase can be inhibited by two types of donor molecules: (i) small inorganic anions such as azide and fluoride, which bind to the trinuclear copper cluster of the enzyme, where the interaction with dioxygen occurs (Messerschmidt, 1997; Casella et al., 1999; Cole, Avigliano, Morpurgo, \& Solomon, 1991; Huang, Sakurai, Maritano, Marchesini, \& Suzuki, 1999; Gromov, Marchesini, Farver, Pecht, \& Goldfarb, 1999), and (ii) organic donor molecules acting as competitive inhibitors toward ascorbate, which have been proposed to bind in a pocket adjacent to the type $1 \mathrm{Cu}$ center (Casella et al., 1999; Gaspard et al., 1997). From the analysis of a Connolly surface for the X-ray structure model of AO, Messerschmidt et al. (1992) identified this pocket as the possible substrate binding site. A recent X-ray crystal study performed on laccase from Trametes versicolor showed that a molecule of the fungus culture inducer (and very slow substrate) 2,5-xylidine remained entrapped in the binding cavity near type $1 \mathrm{Cu}$ (Bertrand et al., 2002). This binding cavity is rather wide $(10 \AA \times 10 \AA \times 20 \AA)$ and the aromatic amine is held in position by hydrophobic interactions with a few protein residues and by hydrogen bonding interactions of the amino group with one of the type $1 \mathrm{Cu}$ His ligands and an Asp residue (Bertrand et al., 2002). The model for the AO-substrate interaction emerging from the present docking investigation shows that the smaller size of the cavity near type 1 $\mathrm{Cu}$ allows the establishment of multiple interactions with ascorbate. Also in this case, one of the type 1 $\mathrm{Cu}$ His ligands is hydrogen bonded to the substrate, but four or five additional residues of the protein are also hydrogen bonded, and a Trp residue completes the set of substrate recognition interactions through stacking with the ascorbate lactone ring. These features make substrate binding to AO very tight and explains the rather high substrate specificity exhibited by this enzyme (Casella et al., 1999; Wimalasena \& Dharmasena, 1994). In addition, participation of the type $1 \mathrm{Cu}$ His ligand in the substrate binding interaction confirms that this residue represents the site of electron entry into the enzyme during the catalytic activity (Bertrand et al., 2002; Gaspard et al., 1997).

Simple phenols can be accommodated within the AO substrate binding site with a disposition similar to that assumed by ascorbate. In particular, not only the phenol molecule is deeply buried into the crevice formed by the active site, as observed for L-ascorbate, but also the relative orientations of the phenyl ring and ascorbate lactone ring are very similar. This result provides a structural basis to the pattern of inhibition effects observed for phenolic compounds. It also accounts for the observation that the inhibitory effect is completely prevented when a substituent of large size is present on the phenol ring, as in p-tert-butylphenol (Gaspard et al., 1997), due to space limitation in the protein cavity. To provide unambiguous experimental support to the location of the inhibitor binding site, paramagnetic ${ }^{19} \mathrm{~F}$ NMR relaxation measurements on AO-fluorophenol and ZnT2-AO-fluorophenol complexes were performed. The investigation of the latter metal substituted AO derivative was necessary to assess the eventual contribution to relaxation by binding of additional inhibitor molecules in relative proximity to type $2 \mathrm{Cu}$. A small effect attributable to this $\mathrm{Cu}$ center actually exists, as shown by comparison of the 
data for AO and ZnT2-AO in Table 1, and is probably due to nonspecific binding of the fluorophenols on the protein surface around the trinuclear cluster. For a thorough interpretation of the data, the small relaxation contribution to the bound phenol nuclei due to the slow tumbling motion of the AO-fluorophenol complexes had to be subtracted. The diamagnetic $\mathrm{Zn}_{4}$-AO derivative was selected for this purpose because it proved to be more resistant to denaturation than apoAO in the presence of the excess amounts of the fluorophenols used in the measurements.

Therefore, the NMR relaxation experiments show that indeed the binding site of the phenolic inhibitors is close to type $1 \mathrm{Cu}$ and far from the trinuclear copper cluster. The $\mathrm{Cu}$-fluorine distances for the bound fluorophenols estimated in this way are about 30\% shorter than those allowed by docking calculations and this certainly reflects the uncertainty in the $\tau_{\mathrm{C}}$ value employed for the enzyme. On the other hand, computational procedures cannot take into account slight conformational rearrangements occurring in the active site on binding of the inhibitor. This is probably the reason for the discrepancy of the two approaches in localizing the position of $p$-fluorophenol within the AO cavity. The docking calculations freeze the disposition of this phenol slightly removed from type $1 \mathrm{Cu}$, by overweighing the hydrogen bonding interaction between the hydroxyl group and the Ser188B residue with respect to that between the fluorine atom and the type $1 \mathrm{Cu}$ His512A ligand. The ${ }^{19} \mathrm{~F}$ relaxation data actually agree with a dynamic equilibrium between two positions for this inhibitor within the AO cavity, because the $\mathrm{Cu}-\mathrm{F}$ distance for $p$-fluorophenol is about $10 \%$ larger than that found for $o$-fluorophenol (Table 1). In conclusion, the present investigation has shown the features that make the active site binding pocket of $\mathrm{AO}$ so specific for ascorbate and given a structural basis for the understanding of the mechanism of AO inhibition by phenolic compounds.

\section{Acknowledgements}

The authors thank the Italian CNR and the University of Pavia (through FAR) for support. Dr. L. Santagostini thanks Consorzio Interuniversitario di Ricerca per la Chimica dei Metalli nei Sistemi Biologici (C.I.R.C.M.S.B) for financial support.

\section{References}

Arrigoni, O. (1994). Ascorbate system in plant development. Journal of Bioenergetics and Biomembranes, 26, 407-419.

Avigliano, L., \& Finazzo-Agrò, A. (1997). In A. Messerschmidt (Ed.), Multicopper oxidases (pp. 251-284). Singapore: World Scientific.

Bertini, I., \& Luchinat, C. (1996). NMR of paramagnetic substances. Coordination and Chemistry Reviews, 150, 111130.

Bertini, I., Briganti, F., Luchinat, C., Mancini, M., \& Spina, G. (1985). The electron-nucleus dipolar coupling in slow rotating systems. 2. The effect of $\mathrm{g}$ anisotropy and hyperfine coupling when $S=1 / 2$ and $I=3 / 2$. Journal of Magnetic Resonance, $63,41-55$.

Bertrand, T., Jolivat, C., Briozzo, P., Caminade, E., Joly, N., Madzak, C., \& Mougin, C. (2002). Crystal structure of a four-copper laccase complexed with an arylamine: insights into substrate recognition and correlation with kinetics. Biochemistry, 41, 7325-7333.

Casella, L., Fantucci, P., Gullotti, M., \& Marchesini, A. (1984). Spectral study of ascorbate oxidase. Inorganica Chimica Acta, 91, 189-194.

Casella, L., Gullotti, M., Pallanza, G., Pintar, A., \& Marchesini, A. (1991). Spectroscopic and binding studies of azide to type-2 copper depleted ascorbate oxidase from zucchini. Biology of Metals, 4, 81-89.

Casella, L., Monzani, E., Santagostini, L., De Gioia, L., Gullotti, M., Fantucci, P., Beringhelli, T., \& Marchesini, A. (1999). Inhibitor binding studies on ascorbate oxidase. Coordination Chemistry Reviews, 185-186, 619-628.

Casella, L., Poli, S., Gullotti, M., Selvaggini, C., Beringhelli, T., \& Marchesini, A. (1994). The chloroperoxidase-catalyzed oxidation of phenols. Mechanism, selectivity and characterization of enzyme-substrate complexes. Biochemistry, 33, 63776386.

Cole, J. L., Avigliano, L., Morpurgo, L., \& Solomon, E. I. (1991). Spectroscopic and chemical studies of the ascorbate oxidase trinuclear copper active site: Comparison to laccase. Journal of American Chemical Society, 113, 9080-9089.

Cordoba, F., \& Gonzales-Reyes, J. A. (1994). Ascorbate and plant cell growth. Journal of Bioenergetics and Biomembranes, 26, 399-405.

Dewar, M. J. S., Zoebisch, E. G., Healy, E. F., \& Stewart, J. J. P. (1985). Development and use of quantum mechanical molecular models. 76. AM1: A new general purpose quantum mechanical molecular model. Journal of the American Chemical Society, 107, 3902-3909.

Fraterrigo, T. L., Miller, C., Reinhammar, B., \& McMillin, D. R. (1999). Which copper is paramagnetic in the type 2/type 3 cluster of laccase? Journal of Biological Inorganic Chemistry, 4, 183-187.

Gaspard, S., Monzani, E., Casella, L., Gullotti, M., Maritano, S., \& Marchesini, A. (1997). Inhibition of ascorbate oxidase by phenolic compounds. Enzymatic and spectroscopic studies. Biochemistry, 36, 4852-4859. 
Gromov, F., Marchesini, A., Farver, O., Pecht, I., \& Goldfarb, D. (1999). Azide binding to the trinuclear copper center in laccase and ascorbate oxidase. European Journal of Biochemistry, 266, $820-830$.

Hakulinen, N., Kiiskinen, L. L., Kruus, K., Saloheimo, M., Paananen, A., Koivula, A., \& Rouvinen, J. (2002). Crystal structure of a laccase from Melanocarpus albomyces with an intact trinuclear copper site. Nature Structural Biology, 9, 601605.

Huang, H.-W., Sakurai, T., Maritano, S., Marchesini, A., \& Suzuki, S. (1999). EPR and magnetic susceptibility studies of the trinuclear copper center in native and azide-reacted zucchini ascorbate oxidase. Journal of Inorganic Biochemistry, 75, 1925.

Kau, L.-S., Spira-Solomon, D. J., Penner-Hahn, J. E., Hodgson, K. O., \& Solomon, E. I. (1987). X-ray absorption edge determination of the oxidation state and coordination number of copper. Application to the type 3 site in Rhus vernicifera laccase and its reaction with oxygen. Journal of the American Chemical Society, 109, 6433-6442.

Kroneck, P. M. H., Armstrong, F. A., Merkle, H., \& Marchesini, A. (1982). Ascorbate oxidase: Molecular properties and catalytic activity. Advances in Chemistry Series, 200, 223-248.

Li, J.-B., McMillin, D. R., \& Antholine, W. E. (1992). Evidence for temperature-dependent changes in the coupling within the type 2/type 3 cluster of laccase. Journal of the American Chemical Society, 114, 725-727.

Lindley, P., Zaitseva, I., Zaitsev, V., Card, G., \& Moshkov, K. (1997). In B. Box, \& A. Messerschmidt (Eds.), Multicopper oxidases (pp. 81-102). Singapore: World Scientific.

Marchesini, A., \& Kroneck, P. M. H. (1979). Ascorbate oxidase from Cucurbita pepo medullosa. New method of purification and reinvestigation of properties. European Journal of Biochemistry, 101, 65-76.

Marchesini, A., Cappelletti, P., Canonica, L., Danieli, B., \& Tollari, S. (1977). Evidence about the catecholoxidase activity of the enzyme ascorbate oxidase extracted from Cucurbita pepo medullosa. Biochimica et Biophysica Acta, 484, 290-300.

Messerschmidt, A. (1997). Spatial structures of ascorbate oxidase, laccase and related proteins: Implications for the catalytic mechanism. In A. Messerschmidt (Ed.), Multicopper oxidases (pp. 23-79). Singapore: World Scientific.

Messerschmidt, A., Ladenstein, R., Huber, R., Bolognesi, M., Avigliano, L., Petruzzelli, R., Rossi, A., \& Finazzi-Agrò, A. (1992). Refined crystal structure of ascorbate oxidase at $1.9 \AA$ resolution. Journal of Molecular Biology, 224, 179-205.
Messerschmidt, A., Luecke, H., \& Huber, R. (1993). X-ray structures and mechanistic implications of three functional derivatives of ascorbate oxidase from zucchini. Reduced, peroxide and azide forms. Journal of Molecular Biology, 230, 997-1014.

Messerschmidt, A., Rossi, A., Ladenstein, R., Huber, R., Bolognesi, M., Gatti, G., Marchesini, A., Petruzzelli, R., \& Finazzi-Agrò, A. (1989). X-ray crystal structure of the blue oxidase ascorbate oxidase from zucchini. Analysis of the polypeptide fold and a model of the copper sites and ligands. Journal of Molecular Biology, 206, 513-529.

Redaelli, C., Monzani, E., Santagostini, L., Casella, L., Sanangelantoni, A. M., Pierattelli, R., \& Banci, L. (2002). Characterization and peroxidase activity of a myoglobin mutant containing a distal arginine. ChemBioChem, 3, 226-233.

Reinhammar, B., \& Malmström, B. G. (1981). In T. G. Spiro (Ed.), Copper proteins (pp. 109-150). New York: Wiley.

Segel, I. H. (1975). Enzyme kinetics. New York: Wiley.

Solomon, I., \& Bloembergen, N. (1956). Nuclear magnetic interactions in the HF molecule. Journal of Physical Chemistry, 25, 261-266.

Solomon, E. I., Sundaram, U. M., \& Machonkin, T. E. (1996). Multicopper oxidases and oxygenases. Chemical Reviews, 27, 2563-2605.

Van Gunsteren, W. F., \& Berendsen, H. J. C. (1990). Molecular dynamics computer simulation. Method, application and perspectives in chemistry. Angewandte Chemie International Edition in English, 29, 992-1023.

Vold, R. L., Waugh, J. S., Klein, M. P., \& Phelps, D. E. (1968). Measurement of spin relaxation in complex systems. Journal of Chemical Physics, 48, 3831-3832.

Wheeler, G. L., Jones, M. A., \& Smirnoff, N. (1998). The biosynthetic pathway of vitamin $\mathrm{C}$ in higher plants. Nature, 393, 365-369.

Williams, T. J., \& Falk, M. C. (1986). Spatial relationship between the copper and carbonyl cofactors in the active site of pig plasma amine oxidase. The Journal of Biological Chemistry, 261, 15949-15954.

Wimalasena, K., \& Dharmasena, S. (1994). Substrate specificity of ascorbate oxidase: unexpected similarity to the reduction site of dopamine $\beta$-monooxygenase. Biochemical and Biophysical Research Communications, 203, 1471-1476.

Zaitseva, I., Zaitsev, V., Card, G., Moshkov, K., Box, B., Ralph, A., \& Lindley, P. (1996). The X-ray structure of human serum ceruloplasmin at $3.1 \AA$ A: Nature of the copper centers. Journal of Biological Inorganic Chemistry, 1, 15-23. 